\title{
CONSTRUCCIÓN Y VALIDACIÓN DEL INVENTARIO DE RELACIONES INTERPERSONALES Y DEPENDENCIAS SENTIMENTALES (IRIDS-100)
}

\section{CONSTRUCTION AND VALIDATION INVENTORY SENTIMENTAL RELATIONSHIPS AND DEPENDENCIES (IRIDS-100)}

Carlos Sirvent Ruiz ${ }^{1}$ y María de la Villa Moral Jiménez²

${ }^{1}$ Fundación Instituto Spiral, España

²Universidad de Oviedo, España

\section{Abstract}

This article presents the validation of the IRIDS-100 or Inventory of Interpersonal Relationships and Affective Dependencies, the latter being subdivided into 1) Emotional Dependency (maladaptive behavior contingent to an affective-dependent interrelationship), 2) Codependency (caregiver-heterocontrol syndrome) and 3) Bidependency (affective dependency engrafted on a substance dependency). The purpose of this paper is to construct and validate the Inventory of Interpersonal Relationships and Sentimental Dependencies (IRIDS-100). The total sample was made up of 880 individuals, 100 of them (11.4\%) identified as emotional dependent; $52(\mathrm{n}=$ $5.9 \%)$ as codependent, and $75(n=8.5 \%)$ as bidependent. 311 (35.3\%) general population individuals and various comparison samples (addicts and non-codependent relatives) were selected via simple random sampling. AFE (principal component extraction method) was used, and reliability, internal consistency, and content and decision validity criteria were assessed. IRIDS-100 psychometric guarantees were confirmed, and a factor structure made up of seven explicative dimensions was found: Dependent Triad, Accommodation, Self-deception, Negative Feelings, Personal History, Characterosis, and Codependent Triad. Analyzed constructs have entity and rationale owing to their social and health impact: $8.66 \%$ of the general population (control group) has a remarkable relationship dependency level. We propose to go dig into the relationship dependency phenomenon and its associated factors due to their double evaluation and therapeutic utility.

Keywords: Relationships, sentimental dependence, emotional dependency, codependency, bidependency.

\section{Resumen}

El propósito de este trabajo es construir y validar el Inventario de Relaciones Interpersonales y Dependencias Sentimentales (IRIDS-100) que evalúa las Dependencias Relacionales. La muestra total está integrada por 880 sujetos, de los cuales 100 (11,4\%) están filiados como dependientes emocionales; 52 ( $n=5,9 \%)$ como codependientes y 75 ( $n=8,5 \%)$ como bidependientes. Se han seleccionado mediante un muestreo aleatorio simple $311(35,3 \%)$ sujetos de población general y varias muestras de comparación (adictos y familiares no codependientes). Se ha empleado un AFE (método de extracción de componentes principales) y se evaluaron criterios de confiabilidad, consistencia interna y validez de contenido y decisión. Se han confirmado las garantías psicométricas del IRIDS-100 y se ha hallado una estructura factorial integrada por siete dimensiones explicativas: Triada Dependiente, Acomodación. Autoengaño, Sentimientos Negativos, Caracterosis, Antecedentes personales y Triada codependiente. Los constructos analizados poseen entidad y razón de ser por el impacto sociosanitario de los mismos: un 8,66\% de la población general (grupo control) presenta un nivel notable de dependencia relacional. Proponemos profundizar sobre el fenómeno de las dependencias relacionales y factores asociados por su doble utilidad evaluativa y terapéutica.

Palabras clave: Relaciones interpersonales, dependencia sentimental, dependencia emocional, codependencia, bidependencia.

Correspondencia: María de la Villa Moral Jiménez

Facultad de Psicología, Plaza Feijóo, s/n - Despacho 211

33003 Oviedo

mvilla@uniovi.es 
Etimológicamente, el concepto dependencia relacional define el conjunto de comportamientos adictivos cuya matriz nuclear es la relación interpersonal, pudiéndose considerar la naturaleza psicopatológica de la misma como un comportamiento desadaptativo contingente a una interrelación afectivodependiente (Sirvent et al., 2004). A efectos ilustrativos, nos referiremos al mismo proceso cuando empleemos indistintamente las expresiones dependencia sentimental, dependencia afectiva, dependencia emocional y adicción al amor, esta última de amplio uso en la literatura americana. Sin embargo, la codependencia y la bidependencia son conceptos diferentes que matizaremos a lo largo del artículo.

¿Por qué hacer una investigación sobre relaciones interpersonales y dependencias sentimentales? El primer gran argumento que justificaría profundizar en este ámbito sería la elevada morbilidad de estos trastornos. La mayoría de investigadores sitúan la prevalencia de la adicción al amor entre el 3 y el $6 \%$ de la población adulta en general (Freimuth et al., 2008; Krueger, \& Kaplan, 2001; Kuzma, \& Black, 2008; Sussman, 2010; Timmreck, 1990), de modo que estudios con jóvenes y universitarios elevan los porcentajes al 11,9\% (Mac Laren, \& Major, 2010) e incluso el 25,9\% (Cook, 1987). Respecto a su prevalencia en población general española en el estudio de Jiménez Burillo, Sangrador, Barrón y Yela (1995) se declararon como interdependientes sentimentales el $55 \%$ de los participantes $(\mathrm{N}=1949)$, resultados similares a los obtenidos por Caldera y Tascón (2001) sobre 100 universitarios, en los que se hallaron un 54\% de casos. En estudios realizados por Sirvent, Moral, Blanco y Palacios (2004) se baraja que un $12 \%$ de la población general presenta dependencia emocional y un $8,66 \%$ lo padece de forma severa, si bien se declara como dependiente emocional el 45,8\% de los participantes (Sirvent et al., 2004). En población juvenil representa una problemática asociada a la violencia en el noviazgo constatándose que los jóvenes victimizados presentan mayor dependencia emocional (Moral, García, Cuetos y Sirvent, 2017).

La dependencia emocional fue descrita por Scott (1978) según los siguientes rasgos clínicos: voracidad por la persona de la que se depende, experimentación de un vacío interior que nunca se llena, insatisfacción permanente con la sensación de que algo les falta, intolerancia a la soledad, cambios emocionales y carencia de un verdadero sentido de la propia identidad.
La codependencia describe un estilo disfuncional de relacionarse con los demás (Irwin, 1995). Según Hands y Dear (1994) los criterios que definen la codependencia son: $1^{\circ}$ ) intentos fallidos por controlarse a uno mismo y a los demás, $2^{\circ}$ ) toma excesiva de responsabilidades sobre las necesidades de los otros hasta el punto de la autonegligencia, $3^{\circ}$ ) distorsión de las fronteras entre la separación y la intimidad y $4^{\circ}$ ) relaciones interpersonales inescapables. La codependencia afecta más a mujeres, sobre todo a parejas de adictos o alcohólicos (Coleman, 1987), aunque también a cuidadores y profesionales, demasiado volcadas en el paciente (Fausel, 1988).

El tercer subtipo de dependencia relacional, la bidependencia, es un constructo que significa dependencia sobre dependencia o, lo que es lo mismo, una dependencia afectiva muy peculiar y de notables características sociopáticas, con búsqueda de sensaciones e impulsividad como elementos definitorios (Sirvent, 1999) instalada en un sujeto adicto a sustancias. Es mucho más frecuente en mujeres, llegando a suponer hasta un $65 \%$ del total de mujeres adictas que mantienen una relación de pareja.

La evaluación de las relaciones interpersonales es compleja, no sólo por la dificultad de definición operacional del propio constructo, sino por la escasez de instrumentos de evaluación que cuenten con las garantías psicométricas exigibles. Una de las pruebas más conocidas es el Interpersonal Dependence Inventory (IDI, Hirschfeld et al., 1977) que se refiere a la dependencia interpersonal como un conjunto de pensamientos, creencias, sentimientos y comportamientos que giran en torno a las necesidades de asociarse estrechamente a otras personas. La Peer Attachment Interview (Bartholomew, \& Horowitz, 1991) es una entrevista semiestructurada que valora la importancia de las relaciones íntimas considerando entre otros aspectos los conflictos, el afecto, la vida social, la dependencia, los celos, la confianza, y los sentimientos en la relación (Scharfe, \& Bartholomew, 1994). La Relationship Profile Test (RPT) (Bornstein, \& Languirand, 2003) consiste en un autoinforme de 30 ítems con tres subescalas: dependencia destructiva, dependencia saludable y desprendimiento disfuncional. Asimismo, otro de los inventarios de interés es la Trait-Specific Dependence Inventory (TSDI) (Ellis et al., 2002) con seis escalas basadas en la hipótesis de la dependencia inerpersonal como rasgo de la personalidad. 
En la literatura científica hay numerosos estudios que correlacionan el binomio "apego-cuidados" con la satisfacción dentro de las relaciones y el apoyo social (Madey, \& Rodgers, 2009; Sternberg, 2013). Uno de los estudios más interesantes lo efectúa Masuda (2003) correlacionando la satisfacción dentro de la relación con dos tipos de amor (erótico y de compañero), para lo cual seleccionó 33 estudios destacando la Passionate Love Scale (PLS) de Hatfield y Sprecher (1986) y la Rubin's Love Scale and Rubin's Liking Scale (Rubin, 1970), concluyendo que la satisfacción correlacionaba inequívocamente con el denominado amor erótico, no así con el llamado amor de compañero.

Respecto a la codependencia, la Escala de Codependencia Composite (CCS) (Marks et al., 2012) es una medida de 19 ítems diseñada para evaluar rasgos codependientes en base a tres factores: auto-sacrificio, control interpersonal, y supresión emocional. Otros instrumentos conocidos son el CdQ de 36 ítems de Roehling y Gaumond (1996), el CODAT de HughesHammer (1998) y el Spann-Fischer (1991) que cuenta con la ventaja de estar más actualizado.

En suma, dada nuestra conceptualización de las dependencias relacionales como trastornos con base adictiva afectivodependiente y cuya la base etiológica no se fundamenta en exclusividad sobre lo caracterológico, el objetivo general de esta investigación consiste en la construcción y validación de un instrumento válido y fiable para medir estas dependencias y sus factores asociados, para así proporcionar al clínico un instrumento de utilidad diagnóstico-evaluativa y terapéutica.

Los objetivos específicos propuestos serían:

a) Explorar los perfiles sintomáticos descriptivos de los constructos Dependencia Sentimental (DS), Codependencia (CDP) y Bidependencia (BDP) en cada una de las dimensiones y factores sintomáticos explorados.

b) Analizar la existencia de diferencias significativas en las puntuaciones medias de las correspondientes subescalas y factores explorados entre las subpoblaciones clínicas y la población control.

c) Ampliar la explicación con la propuesta de un modelo explicativo de las dependencias sentimentales en base a las características intrínsecas del instrumento aquí expuesto (subescalas y factores sintomáticos) de clara orientación clínica.
Como hipótesis de estudio se plantea que se hallará un perfil sindrómico indicativo de mayor patología en la subpoblación clínica respecto a la población general y en relación a las muestras de comparación (adictos y familiares no codependientes) en las correspondientes escalas y factores sintomáticos. Se prevé que se hallarán diferencias significativas intramuestrales en las subpoblaciones clínicas, de acuerdo al perfil de dependencia sentimental descrito.

\section{MÉTODO}

\section{Participantes}

En esta investigación se empleó un diseño no experimental transversal cuantitativo de tipo descriptivo correlacional (Hernández, Fernández, \& Baptista, 1998). Se han seleccionado muestras clínicas y de comparación mediante un diagnóstico llevado a cabo por el equipo multidisciplinar de psicoterapeutas (psicólogos, médicos y psiquiatra) de la Fundación Instituto Spiral en Oviedo y Madrid y por profesionales externos mediante acuerdo inter-jueces. La muestra total está integrada por 880 sujetos, de los cuales el $11.4 \%(n=100)$ están filiados como dependientes emocionales (DE), el 5.9\% ( $n=52)$ como codependientes (CDP) y el $8.5 \% \quad(n=75)$ como bidependientes (BDP). Asimismo, han sido seleccionados mediante un muestreo aleatorio simple de la población del Principado de Asturias 311 sujetos de población general que representan el $35.3 \%$ de la muestra total. Como grupos de comparación se han seleccionado 295 adictos en tratamiento en la Fundación Instituto Spiral y 47 familiares no codependientes, que representan, respectivamente el $33.5 \%$ y el $5.3 \%$ elegidos mediante un muestreo no probabilístico de tipo incidental. Todos los participantes firmaron un acuerdo de consentimiento informado y se les garantizó en todo momento el uso exclusivo de sus respuestas para fines de investigación.

De los cien sujetos diagnosticados como dependientes emocionales, el $73.1 \%$ son mujeres y el $26.9 \%$ restante hombres. De acuerdo con la estratificación por edades, la media se halla en 38.84 años ( $D E=9.554)$ con un rango de edad de 20 a 61 años. Casi la mitad de los mismos están solteros (44.9\%) y más de un tercio declara haber tenido dos matrimonios/parejas (33.3\%). Se manifiesta que el 16,9\% está diagnosticado de depresión y ansiedad, un 9.9\% de depresión, un $8.5 \%$ de ansiedad, así como respecto al 
historial de drogodependencias el tabaquismo (5.7\%), el consumo de psicofármacos (4.3\%) y el uso de alcohol y cocaína $(2.9 \%)$ representan las principales adicciones.

Se han seleccionado 52 casos de codependientes de la muestra de familiares de pacientes en tratamiento (79.5\% mujeres y $20.5 \%$ hombres, media de edad 49.4 años). El setenta por ciento de los codependientes que integran la muestra están casados y un 16.0\% ( $n=10)$ separados. En relación a sus experiencias de pareja un porcentaje del $61.3 \%(n=31)$ declaran tener/haber tenido la experiencia de compartir su vida con una única pareja.

La subpoblación de bidependientes está integrada por 75 sujetos diagnosticados, con edades comprendidas entre los 17 a 55 años (media=36.87 años), de los cuales el $55.6 \%(n=35)$ son varones. Casi un tercio (30.6\%) con un historial de al menos tres parejas con las que se ha convivido.

En la muestra de población general, de acuerdo a la adscripción por género, un $53.1 \%$ son mujeres $(46.9 \%$ varones), con edades comprendidas entre los 18 y los 73 años (media=36.06, $D E=12.154$ ), predominantemente solteros (67.9\%, $\mathrm{n}=209)$, con estudios universitarios cursados (Grado Medio, 19.7\%, n=61; Grado Superior, $25.5 \%, \mathrm{n}=78$ ) y que han convivido a lo largo de su vida con una $(40.9 \%, n=124)$ o dos parejas $(28.1 \%, n=85)$.

La subpoblación de adictos (no bidependientes) incluye todos los adictos en tratamiento actualmente en la Fundación Instituto Spiral en el momento de realizar el estudio. Relativo a las características sociodemográficas el $64.9 \% \quad(n=121)$ son varones, presentan edades comprendidas entre los 17 y los 56 años, situándose la media en 35.55. El estado civil de soltero es el más representativo $(67.6 \%)$ y el $22.6 \%(n=66)$ de los adictos que integran la muestra han tenido tres o más parejas.

Mediante un acuerdo interjueces se han seleccionado 47 casos de familiares no codependientes del grupo de familiares de adictos en tratamiento (media $=47.91$ años), de los cuales el 53.2\% son mujeres. Un porcentaje muy elevado del $71.8 \%(n=34)$ declaran tener/haber tenido la experiencia de compartir su vida con una única pareja, lo cual también resulta sumamente interesante a efectos de los objetivos planteados (véase Tabla 1).

\section{Instrumentos}

Mediante el test autoaplicado informatizado Inventario de Relaciones Interpersonales y Dependencias Sentimentales (I.R.I.D.S.-100) se evalúan no solo los diferentes tipos de dependencia sentimental existentes, sino escalas y factores de importancia clínica en el ámbito relacional. Consta de ítems evaluados mediante escala Likert de cinco puntos (Muy de acuerdo a Muy en desacuerdo), con adecuadas garantías psicométricas. Como cuestionarios de anclaje se han empleado el Inventario de Dependencias Interpersonales (I.D.I.) de Hirschfeld et al. (1997), el cual consta de 48 ítems, validado en una muestra de 187 sujetos, 121 población general y 66 sujetos con psicopatología psiquiátrica. Obtuvieron un alfa de Cronbach superior a 0.80 en la subescala de dependencia emocional en otra persona, superior a 0.7 para falta de autoconfianza social y superior a 0.70 en aserción de autonomía. También se ha empleado el Codependency Assessment Tool (CODAT) de Hughes-Hammer, Martsolf, \& Zeller (1998) adaptado por Pérez y Delgado (2003) que consta de 45 ítems divididos en tres subescalas: focalización en el otro/negligencia de sí mismo (17 ítems), no afrontamiento (14 ítems) y sobrecontrol (14 ítems), obteniéndose un alfa de Cronbach superior a 0,70 en todas las subescalas. Asimismo, se ha empleado la escala de bidependencia del Test de dependencias Sentimentales (TDS-100) de Sirvent \& Moral (2007) que también cuenta con adecuadas garantías psicométricas (alfa de Cronbach superior a $0,90)$.

\section{Procedimiento}

Inicialmente, se realizó un estudio de pilotaje a objeto de contar con un amplio conjunto de ítems que fueran representativos del constructo de medida. En primer lugar, se efectuó una exhaustiva revisión de la temática de las dependencias sentimentales en la literatura al uso, tanto de la fundamentación teórica como de los instrumentos de análisis. Dos expertos en la materia participaron en el proceso de elaboración de los ítems y construcción del instrumento con el asesoramiento de expertos en Metodología de Ciencias del Comportamiento. Para llevar a cabo la evaluación psicométrica del IRIDS-100 se realizó primeramente la evaluación cualitativa de la batería de ítems que fue sometida a un nuevo proceso de revisión mediante juicio de expertos familiarizados con el constructo a evaluar y que juzgaron la dimensión de pertinencia de cada uno de los ítems y valoraron en una escala Likert la redacción de cada ítem, en cuanto a si éstos resultaban comprensibles. 
Este procedimiento a través del juicio de expertos contribuye notablemente a incrementar la representatividad de los ítems (Carretero-Dios y Pérez, 2007). A dicho efecto se realizó un estudio de investigación en las ciudades de Madrid y Oviedo elaborándose un pre-test y dos tests ulteriores refinados con los síntomas más significativos (mayores saturaciones de los ítems) después de una doble decantación: clínica y experimental.

\section{Análisis de datos}

Con la muestra definitiva se ha analizado la validez de criterio y de constructo del instrumento y se ha revisado la confiabilidad de la escala para medir la consistencia interna a través del coeficiente " $r$ " de Spearman-Brown y estudiada a través del coeficiente de fiabilidad alfa de Cronbach. La validez del constructo se ha analizado por medio de las medidas estadísticas de los elementos, correlación entre elementos y entre elemento y escala total. El análisis de validez de grupos contrastados o de criterio se realizó a través de la comparación de medias, por medio del estadístico ANOVA, una vez confirmado el empleo de estadística paramétrica al comprobarse los supuestos de normalidad y homocedasticidad mediante la prueba de Kolmogorov-Smirnov. Para el Análisis Factorial Exploratorio se empleó el método de componentes principales con posterior rotación ortogonal Varimax, previa verificación de bondad de ajuste a este análisis (índice de determinación, índice Kaiser-Meyer-Olkin y prueba de esfericidad de Barlett).

La recogida de la información se ha llevado a cabo por profesionales dentro del programa terapéutico contando con suficientes garantías metodológicas (asignación de un código identificativo, confidencialidad, etc.). Dado el carácter longitudinal del estudio continúa el seguimiento de la evolución de las poblaciones clínicas (dependientes emocionales, codependientes y bidependientes). El procesamiento y tratamiento estadístico de los datos se ha llevado a cabo mediante el programa SPSS versión 21.0.

Tabla 1. Variables sociodemográficas por tipo de subpoblación

\begin{tabular}{|c|c|c|c|c|c|c|}
\hline \multirow[t]{2}{*}{ Datos sociodemográficos } & $\mathrm{DE}$ & BDP & CDP & $\begin{array}{c}\text { Población } \\
\text { General }\end{array}$ & Adictos & $\begin{array}{c}\text { Familiares no } \\
\text { CDP }\end{array}$ \\
\hline & $\% \mathrm{n}$ & $\% \mathrm{n}$ & $\% \mathrm{n}$ & $\% \mathrm{n}$ & $\% \mathrm{n}$ & $\% \mathrm{n}$ \\
\hline \multirow[t]{2}{*}{ Género } & Femenino & Masculino & Femenino & Femenino & Masculino & Femenino \\
\hline & $73.1(57)$ & $55.6(35)$ & $79.5(31)$ & $53.1(173)$ & $64.9(191)$ & $53.2(25)$ \\
\hline \multirow[t]{4}{*}{ Edad } & 20 a 61 & 17 a 55 & 22 a 73 & 17 a 62 & 17 a 65 & 25 a 63 \\
\hline & - & - & - & - & - & - \\
\hline & X 38.84 & X 36.87 & X 49.40 & X 38.84 & X 37.75 & X 47.91 \\
\hline & (DT 9.554) & (DT 9.722) & (DT 11.598) & (DT 12,154) & (DT 9.554) & (DT 10.92) \\
\hline \multirow[t]{2}{*}{ Estado Civil } & Soltero & Soltero & Casado & Soltero & Soltero & Casado \\
\hline & $44.9(35)$ & $67.6(50)$ & $70.0(36)$ & $67.9(209)$ & $59.6(167)$ & $72.2(34)$ \\
\hline \multirow[t]{2}{*}{ Profesión } & Funcionario & $\begin{array}{c}\text { Obrero } \\
\text { cualificado }\end{array}$ & $\begin{array}{c}\text { Labores } \\
\text { domésticas }\end{array}$ & $\begin{array}{c}\text { Obrero } \\
\text { cualificado }\end{array}$ & $\begin{array}{l}\text { Obrero no } \\
\text { cualificado }\end{array}$ & $\begin{array}{c}\text { Labores } \\
\text { domésticas }\end{array}$ \\
\hline & $27(20)$ & $28.6(21)$ & $45.6(23)$ & $19.9(61)$ & $36.5(107)$ & $32.9(15)$ \\
\hline \multirow[t]{2}{*}{ Nivel cultural } & Bachiller & Bachiller & Primarios & Bachiller & Primarios & Primarios \\
\hline & $25.3(19)$ & $27.0(20)$ & $24.0(14)$ & $27.7(84)$ & $37.5(110)$ & $21.0(10)$ \\
\hline \multirow[t]{2}{*}{ Nivel socioeconómico } & Medio & Medio & Medio & Medio & Medio & Medio \\
\hline & $72.1(44)$ & $81.8(61)$ & $80.7(41)$ & $84.2(202)$ & $65.2(184)$ & $79.8(37)$ \\
\hline \multirow[t]{2}{*}{$N^{\circ}$ de miembros en el hogar } & 2 & 3 & 4 & 4 & 4 & 4 \\
\hline & $24.4(18)$ & $35.3(26)$ & $36.2(18)$ & $42.3(130)$ & $27.8(82)$ & $37.7(18)$ \\
\hline \multirow[t]{2}{*}{$N^{\circ}$ de hijos } & 0 & 0 & 1 & 0 & 0 & 2 \\
\hline & $41.7(30)$ & $45.7(34)$ & $31.3(1)$ & $55.4(165)$ & $47.3(139)$ & $38.7(19)$ \\
\hline \multirow[t]{2}{*}{$\mathrm{N}^{\circ}$ de hermanos } & 2 & 3 & 3 & 2 & 3 & 3 \\
\hline & $36.6(26)$ & $34.4(25)$ & $37.2(18)$ & $40.0(124)$ & $32.7(96)$ & $35.2(16)$ \\
\hline \multirow[t]{2}{*}{$\mathrm{N}^{\circ}$ de parejas } & 2 & 3 & 1 & 1 & 3 & 1 \\
\hline & $33.3(24)$ & $30.6(230)$ & $61.3(37)$ & 40.9 (124) & $22.6(66)$ & $71.8(34)$ \\
\hline
\end{tabular}




\section{RESULTADOS}

\section{Confiabilidad}

Se ha llevado a cabo un análisis factorial exploratorio para estudiar la dimensionalidad de la muestra. De forma previa se ha calculado la medida de adecuación muestral de Kaiser-Meyer-Olkin (KMO) con un valor de 0.815 y el test de esfericidad de Barlett resultando estadísticamente significativo (.00), lo cual indica que las variables se relacionaban entre sí, con un valor del estadístico $\mathrm{Chi}^{2}=3290.888 ; \mathrm{p}<0.0001$. La escala cuenta con los requisitos mínimos que garantizan la pertinencia del Análisis de Componentes Principales y en primera instancia se ha empleado la rotación Varimax. A pesar de que el procedimiento de rotación usado se aconseja para casos en los que los factores no están relacionados, se optó por éste debido al interés teórico de los investigadores de separar en la medida de lo posible, los factores resultantes, criterio contemplado en el análisis de Carretero-Dios y Pérez (2007), quienes indican que con un número de ítems superior a 20, la corroboración de que existe una adecuada intercorrelación entre ellos, y con muestras de participantes de al menos 300, las diferencias entre las soluciones factoriales proporcionadas por distintos métodos son despreciables (Snook, \& Gorsuch, 1989) y se recomienda el uso del análisis de componentes principales (Cortina, 1993) y la rotación ortogonal Varimax (véase Comrey, 1988).

El Inventario está integrado por 7 Dimensiones o factores explicativos (Triada Dependiente, Acomodación, Autoengaño, Sentimientos Negativos, Caracterosis, Antecedentes personales y Triada codependiente), que explican el 53.72\% de la varianza total (índices de adecuación muestral $\mathrm{KMO}=0.942$ y $\mathrm{X}^{2}=33752.671 ; \mathrm{p}$ $<0.000$ ), de los cuales el primer factor Triada Dependiente, que explora dependencia, búsqueda de sensaciones y craving, explica el $24.13 \%$. Asimismo, se ha demostrado la probada eficacia clínica de 23 factores sindrómicos creados por acuerdo inter-jueces y que han demostrado su validez discriminante entre las subpoblaciones clínicas, población general y submuestras de comparación (Tabla 2).

La confiabilidad interna total del IRIDS-100 fue muy elevada, hallándose en el indicador Alfa de Cronbach un valor de .971 para una muestra total de 880 sujetos. La escala ha mostrado ser consistente internamente, dados los valores hallados para cada una de la subescalas, de modo que se puede aseverar que los elementos son homogéneos y que la escala mide de forma consistente la característica para la cual fue elaborada. Los valores oscilan entre el nivel para la Dimensión Sentimientos Negativos (.896) y la obtenida en Caracterosis (.825). La prueba de no aditividad de Tukey es significativa ( $\mathrm{F}=82.401, \mathrm{p}<.0001)$, así como la prueba T cuadrado de Hotelling $(F=5406.948, p<.0001)$.

A continuación se especifican las siete dimensiones que definen el constructo Dependencia Sentimental y su correspondiente descripción clínica:

Triada dependiente. Integrada por los factores sindrómicos denominados Dependencia (necesidad afectiva patológica que desarrolla una persona respecto a otra), Búsqueda de sensaciones (necesidad de estímulos y experiencias nuevas con el fin de alcanzar un nivel óptimo de excitación) y Craving y Abstinencia (vacío desolador e insoportable ante la ausencia del otro/a, máxime si es por distanciamiento o ruptura).

Acomodación. Representa una tendencia a acoplarse pasivamente a una relación afectiva de pareja.

Autoengaño. Compuesta por el factor Autoengaño (incapacidad para darse cuenta de los efectos adversos de la relación con conciencia del problema equívoca o insuficiente); Manipulación (intento de modificar los auténticos sentimientos de la persona de la que se depende); Reiteración (tendencia a la comisión -una y otra vez- de los mismos fallos con el otro/a) y Mecanismos de negación (negación del problema relacional asociado al no enfrentamiento de la situación).

Sentimientos negativos. Definida por los factores Soledad (o aislamiento social) (percepción y vivencia insufrible de sentimientos negativos y tendencia a aislarse cada vez más de los demás desde que tiene o tenía pareja); Vacío emocional (sensación y sentimiento de vacuidad afectiva o constante insatisfacción que no se cubre con nada); Culpa (exculpación ciega e irracional del otro, autoconvenciéndose de que no es realmente así y/o autoinculpación de los propios errores); Autodestrucción (vivencia autodestructiva de la relación prefiriendo no estar vivo); Inescapabilidad emocional (percepción de sentirse atrapado/a, prisionero o retenido en la relación y sin visos de poder superar el problema) y Recreación de sentimientos negativos (aparición espontánea y frecuente de sentimientos negativos). 
Tabla 2. Estructura factorial del IRIDS-100

\begin{tabular}{|c|c|c|c|c|c|c|c|c|c|c|c|c|c|}
\hline \multicolumn{14}{|c|}{ Dimensiones del Inventario de Relaciones Interpersonales y Dependencias Sentimentales (IRIDS-100) } \\
\hline \multicolumn{2}{|c|}{$\begin{array}{c}\text { Triada } \\
\text { Dependiente/ } \\
\text { Interdependencia }\end{array}$} & \multicolumn{2}{|c|}{ Acomodación } & \multicolumn{2}{|c|}{ Autoengaño } & \multicolumn{2}{|c|}{$\begin{array}{l}\text { Sentimientos } \\
\text { Negativos }\end{array}$} & \multicolumn{2}{|c|}{ Caracterrosis } & \multicolumn{2}{|c|}{$\begin{array}{l}\text { Antecedentes } \\
\text { Personales }\end{array}$} & \multicolumn{2}{|c|}{$\begin{array}{c}\text { Triada } \\
\text { Codependiente/ } \\
\text { Heterocontrol }\end{array}$} \\
\hline Ítems & $R^{2}$ & Items & $R^{2}$ & Items & $R^{2}$ & Items & $R^{2}$ & Items & $R^{2}$ & Items & $R^{2}$ & Items & $R^{2}$ \\
\hline 67 & .737 & 33 & .787 & 79 & .722 & 32 & .787 & 41 & .720 & 78 & .761 & 54 & .777 \\
\hline 88 & .726 & 21 & .716 & 86 & .695 & 68 & .712 & 37 & .697 & 40 & .720 & 10 & .667 \\
\hline 2 & .715 & 10 & .667 & 20 & .690 & 28 & $\begin{array}{l}.667 \\
.631\end{array}$ & 82 & $\begin{array}{l}.694 \\
.674\end{array}$ & 72 & $\begin{array}{l}.695 \\
.686\end{array}$ & 38 & .648 \\
\hline 62 & .710 & 92 & .651 & 39 & .647 & 63 & .622 & 84 & .606 & 46 & .616 & 55 & .641 \\
\hline 58 & .681 & 39 & $\begin{array}{l}.647 \\
.515\end{array}$ & 26 & .645 & 85 & .560 & 87 & .590 & 3 & .460 & 75 & .630 \\
\hline 12 & .654 & 18 & . 417 & 11 & $\begin{array}{l}.618 \\
.547\end{array}$ & 36 & $\begin{array}{l}.532 \\
.529\end{array}$ & 13 & $\begin{array}{l}.546 \\
.529\end{array}$ & 93 & .411 & 16 & .597 \\
\hline 6 & .652 & 35 & & 29 & .542 & 65 & .474 & 99 & .522 & 66 & & 43 & .551 \\
\hline 39 & .647 & & & 71 & .461 & 59 & .424 & 59 & .508 & & & 61 & .466 \\
\hline 26 & .645 & & & 23 & $\begin{array}{l}.449 \\
.408\end{array}$ & 45 & $\begin{array}{l}.422 \\
.392\end{array}$ & 100 & $\begin{array}{l}.455 \\
442\end{array}$ & & & 47 & .453 \\
\hline 15 & .628 & & & 74 & .381 & 42 & .392 .389 & 34 & $\begin{array}{l}.442 \\
.439\end{array}$ & & & 90 & .442 \\
\hline 22 & .622 & & & 81 & .324 & 83 & .381 & 31 & .398 & & & 42 & .424 \\
\hline 70 & .566 & & & 76 & $\begin{array}{l}.310 \\
301\end{array}$ & 80 & $\begin{array}{l}.374 \\
370\end{array}$ & 90 & $\begin{array}{r}.385 \\
361\end{array}$ & & & 52 & .416 \\
\hline 97 & .564 & & & 17 & .296 & 91 & $\begin{array}{l}.370 \\
.323\end{array}$ & 94 & $\begin{array}{l}.361 \\
.331\end{array}$ & & & 96 & .376 \\
\hline 29 & .547 & & & 56 & .284 & 80 & .317 & 51 & .316 & & & 14 & .352 \\
\hline 98 & .546 & & & 4 & .264 & 49 & & 73 & .234 & & & & \\
\hline 27 & .477 & & & 7 & .238 & 44 & & 53 & & & & & \\
\hline 34 & .455 & & & 64 & & 77 & & 60 & & & & & \\
\hline 91 & .392 & & & 69 & & 19 & & 50 & & & & & \\
\hline 73 & .385 & & & 25 & & & & 25 & & & & & \\
\hline 49 & .374 & & & 8 & & & & & & & & & \\
\hline 30 & .343 & & & & & & & & & & & & \\
\hline 9 & .337 & & & & & & & & & & & & \\
\hline 5 & .331 & & & & & & & & & & & & \\
\hline 24 & .305 & & & & & & & & & & & & \\
\hline \multicolumn{14}{|c|}{ Muestra Total } \\
\hline \multicolumn{14}{|c|}{ Media } \\
\hline \multicolumn{2}{|l|}{3.1828} & \multicolumn{2}{|l|}{3.1270} & \multicolumn{2}{|l|}{3.1940} & \multicolumn{2}{|l|}{2.9161} & \multicolumn{2}{|l|}{3.3296} & \multicolumn{2}{|l|}{$3, .341$} & \multicolumn{2}{|c|}{2.9214} \\
\hline \multicolumn{14}{|l|}{$D T$} \\
\hline \multicolumn{2}{|l|}{.70119} & \multicolumn{2}{|l|}{.74375} & \multicolumn{2}{|l|}{.67549} & \multicolumn{2}{|l|}{.77285} & \multicolumn{2}{|l|}{.58866} & \multicolumn{2}{|l|}{.94635} & \multicolumn{2}{|c|}{.60683} \\
\hline Alpha & & & & & & & & & & & & & \\
\hline .892 & & .836 & & .867 & & .896 & & .825 & & .831 & & .870 & \\
\hline
\end{tabular}

Caracterosis. Constituida por los factores Identidad (o disolución de la identidad) (necesidad de vincularse estrechamente a la persona de la que se depende); Límites débiles (tendencia a ceder habitualmente ante el otro, influenciabilidad comportamental); Límites rígidos (intransigencia, no aceptación de lo que otros sugieren); Egoísmo/ egotismo/ egocentrismo en la interrelación (dedicación excesiva atención a uno mismo e intervenir en asuntos ajenos para alcanzar intereses propios vinculados a la relación) y Fortaleza del ego/ control y dominio/ juegos de poder (tendencia a competir por el poder en la relación).
Antecedentes personales. Referido a antecedentes de carencias afectivas, abuso o maltrato físico o psicológico y/o antecedentes familiares y/o personales de abuso de sustancias) y, finalmente,

Triada codependiente. Integrada por Orientación rescatadora/sobreprotección/pseudoaltruismo/abnegación (preocupación excesiva porque el otro esté a gusto y empleo de comportamientos de sobreayuda); Sobrecontrol (intento de ejercer un excesivo control sobre el otro/a responsabilizándose demasiado de la vida de los demás, sobreprotección) y Focalización en el otro y 
desatención o descuido de sí mismo (tendencia a experimentar ansiedad o excesiva preocupación porque el otro/a esté a gusto desatendiendo las propias necesidades).

Dado el carácter esencialmente exploratorio del análisis, en una segunda solución factorial en base al criterio de Catell se extrajeron factores por componentes principales y rotación Oblimin directo, empleando un valor delta igual a cero para un grado de correlación moderado entre los factores sugeridos y obteniéndose para la muestra total según el indicador de adecuación muestral de Kaiser-Meyer-Olkin (KMO) una varianza explicada del $62.598 \%$.

La invarianza de la estructura factorial de la escala se contrastó entre los grupos de dependientes sentimentales (dependientes emocionales, bidependientes y codependientes), con objeto de comprobar si se producía algún tipo de modificación en la estructura factorial de la escala o en el porcentaje de variabilidad explicada. Para ello, se replicó el análisis, de modo que los ítems alcanzaron una adecuada variabilidad explicada y los índices de bondad de ajuste obtenidos en la muestra de dependientes sentimentales, confirmaron la estructura bidimensional de los factores de la escala. De este modo, con base en el criterio Káiser (autovalores en la extracción mayores a 1) se analizó la estructura dimensional, la cual explica el $67.31 \%$ de la varianza total en la muestra de dependientes sentimentales (KMO: .913; Barlett, $\mathrm{p}<.001$ ), con una dimensión integrada por los factores Búsqueda de Sensaciones, Dependencia, Autoengaño y Antecedentes y otra por los factores Caracterosis, Focalización en el otro, Sobrecontrol, Sentimientos adversos y Acomodación.

A continuación, se ofrecen los resultados descriptivos básicos hallados en los factores explorados tanto en muestra clínica (véase Tabla 3) como en población general (véase Tabla 4), con valores de medias más bajas en las dimensiones analizadas en población clínica, indicativo de mayor patología.

Tabla 3. Estadísticos descriptivos básicos de la Población Clínica ( $N=227)$

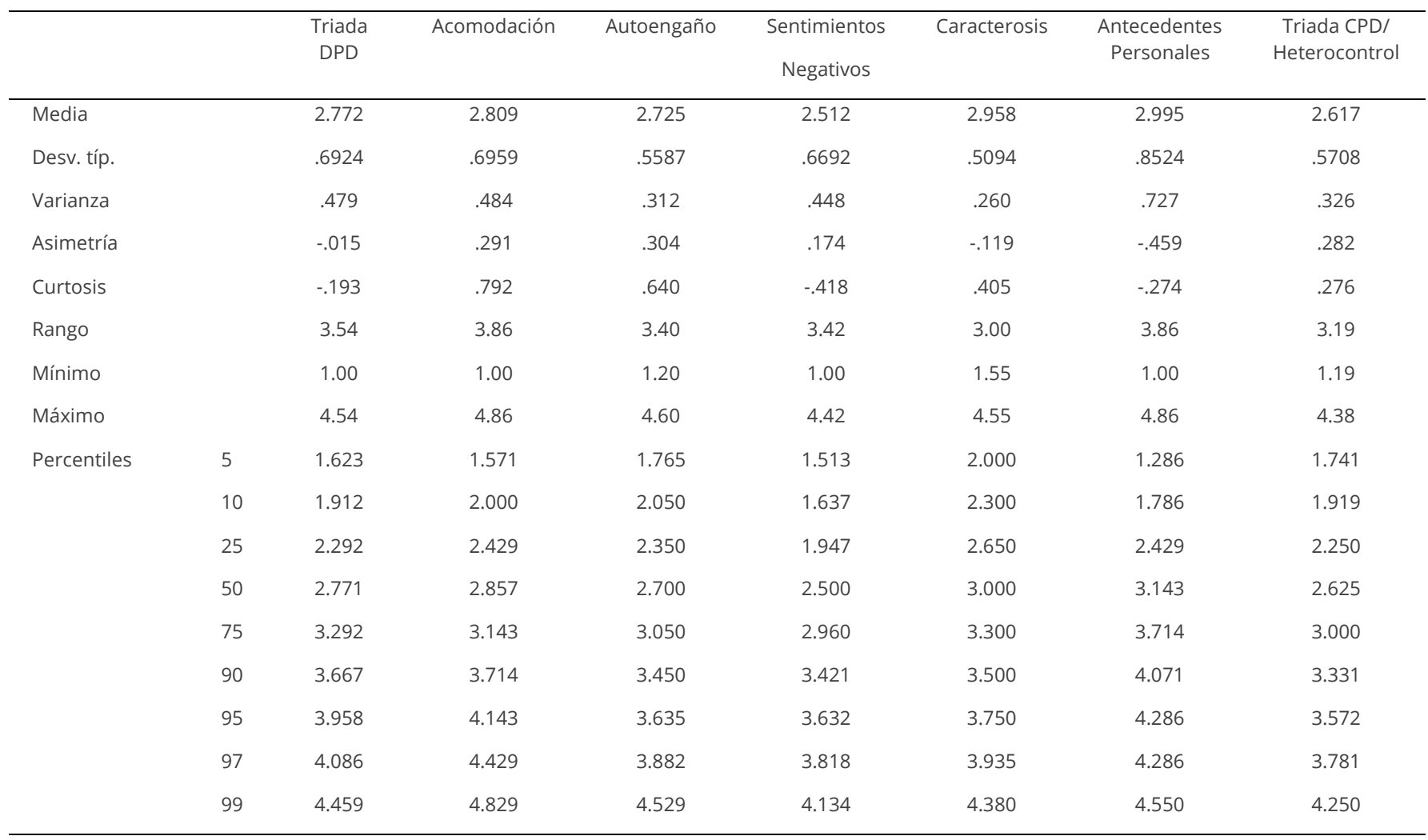

Nota: Triada DDP=Triada Dependiente; Triada CDP=Triada Codependiente 
Tabla 4. Estadísticos descriptivos básicos de la Población General ( $N=311)$

\begin{tabular}{|c|c|c|c|c|c|c|c|c|}
\hline & & Triada DPD & Acomodación & Autoengaño & $\begin{array}{c}\text { Sentimientos } \\
\text { Negativos }\end{array}$ & Caracterosis & $\begin{array}{c}\text { Antecedentes } \\
\text { Personales }\end{array}$ & $\begin{array}{l}\text { Triada CPD/ } \\
\text { Hetercontro }\end{array}$ \\
\hline Media & & 3.413 & 2.903 & 3.463 & 3.1893 & 3.538 & 3.743 & 3.126 \\
\hline Desv. típ. & & .3478 & .2946 & .3206 & .46747 & .2965 & .5662 & .4338 \\
\hline Varianza & & .121 & .087 & .103 & .219 & .088 & .321 & .188 \\
\hline Asimetría & & -.655 & -1.666 & -.902 & -.761 & -.556 & -1.210 & -.615 \\
\hline Curtosis & & .913 & 3.036 & .278 & .106 & -.171 & 1.061 & .332 \\
\hline Rango & & 1.96 & 1.57 & 1.55 & 2.26 & 1.50 & 2.57 & 2.38 \\
\hline Mínimo & & 2.25 & 1.57 & 2.45 & 1.74 & 2.60 & 1.71 & 1.56 \\
\hline Máximo & & 4.21 & 3.14 & 4.00 & 4.00 & 4.10 & 4.29 & 3.94 \\
\hline \multirow[t]{9}{*}{ Percentiles } & 5 & 2.808 & 2.286 & 2.802 & 2.274 & 2.950 & 2.514 & 2.312 \\
\hline & 10 & 2.992 & 2.429 & 2.955 & 2.442 & 3.100 & 3.000 & 2.562 \\
\hline & 25 & 3.250 & 2.714 & 3.250 & 2.895 & 3.350 & 3.429 & 2.875 \\
\hline & 50 & 3.458 & 3.000 & 3.550 & 3.263 & 3.55 & 3.857 & 3.187 \\
\hline & 75 & 3.625 & 3.143 & 3.700 & 3.579 & 3.750 & 4.286 & 3.437 \\
\hline & 90 & 3.833 & 3.143 & 3.800 & 3.684 & 3.900 & 4.286 & 3.687 \\
\hline & 95 & 3.958 & 3.143 & 3.850 & 3.790 & 3.950 & 4.286 & 3.750 \\
\hline & 97 & 4.000 & 3.143 & 3.858 & 3.895 & 4.000 & 4.286 & 3.875 \\
\hline & 99 & 4.163 & 3.143 & 4.000 & 4.000 & 4.050 & 4.286 & 3.937 \\
\hline
\end{tabular}

Nota: Triada DDP=Triada Dependiente; Triada CDP=Triada Codependiente

\section{Consistencia interna}

La matriz de correlaciones inter-elementos entre cada uno de las dimensiones halladas se expone en la Tabla 5. Tal y como se puede comprobar, todas las correlaciones son estadísticamente significativas $(p<.001)$ y superiores a .50. En concreto, las correlaciones más elevadas superiores a .80 se han hallado entre Triada dependiente y Autoengaño $(r=.829)$, así como entre Triada dependiente y Sentimientos negativos $(r=.811)$, entre Autoengaño y Sentimientos negativos $(r=.808)$ así como entre Sentimientos negativos y Caracterosis $(r=.805)$.

Tabla 5. Matriz de correlaciones inter-elementos de las Dimensiones del IRIDS-100

\begin{tabular}{|c|c|c|c|c|c|c|}
\hline & $\begin{array}{c}\text { Triada } \\
\text { Dependiente }\end{array}$ & Acomodación & Autoengaño & $\begin{array}{c}\text { Sentimientos } \\
\text { Negativos }\end{array}$ & Caracterosis & $\begin{array}{c}\text { Antecedentes } \\
\text { Personales }\end{array}$ \\
\hline Triada Dependiente & -- & & & & & \\
\hline Acomodación & $.600 \star \star$ & -- & & & & \\
\hline Autoengaño & $.829 * \star$ & $.640 * \star$ & -- & & & \\
\hline Sentimientos Negativos & $.811 * \star$ & $.621 * \star$ & $.808^{\star \star}$ & -- & & \\
\hline Caracterosis & $.758^{\star *}$ & $.597 * \star$ & $.793^{\star *}$ & $.805^{\star *}$ & -- & \\
\hline Antecedentes Personales & $.518 * \star$ & $.576^{\star \star}$ & $.531 * \star$ & $.529 * \star$ & $.536 * \star$ & -- \\
\hline Triada CPD/ Heterocontrol & $.656^{\star *}$ & $.550 * \star$ & $.699 * *$ & $.758^{* *}$ & $.777 * *$ & $.551 * \star$ \\
\hline
\end{tabular}


La matriz de correlaciones inter-elementos entre cada una de las Escalas (Dependencia Afectiva, Codependencia, Bidependencia y Dependencia Sentimental) que integran el IRIDS-100 se exponen en la Tabla 6.

Tabla 6. Matriz de correlaciones inter-elementos de las Escalas del IRIDS100

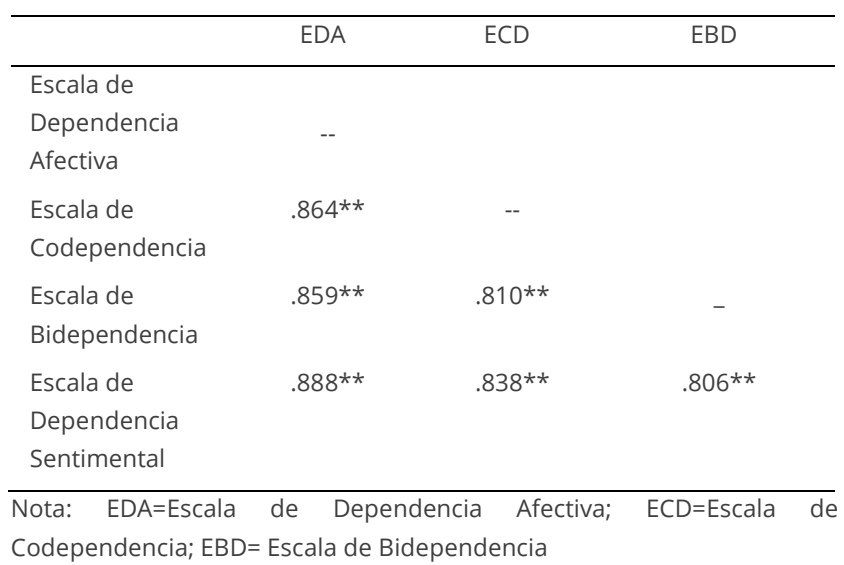

\section{Obtención de evidencias externas de validez}

En este estudio se obtienen evidencias externas de validez dada la trayectoria investigadora en la temática y, a nivel empírico, con el estudio de pilotaje previo del Test de Dependencias Sentimentales (TDS) (Sirvent, \& Moral, 2007) a partir de cuya reformulación surge el actual IRIDS100. Se cumple la validez de criterio al haberse empleado cuestionarios de anclaje, comparándose nuestra situación de medida con los correspondientes estándares ya validados (validez concurrente). En cuanto a la validez discriminante, se comprueba que la medida utilizada no se correlaciona demasiado con las medidas de otros constructos de los que debería diferenciarse.

Por lo que respecta a la validez de decisión se ha efectuado un análisis de comparación de medias. En primer lugar, se exponen valores diferenciales por subtipo de población hallados en la Escala General de Dependencia Sentimental, así como en las Subescalas de Dependencia Emocional, Codependencia y Bidependencia y se constata la existencia de diferencias significativas $(p<.0001)$ en la dirección predicha. En la Escala General de Dependencia Sentimental las subpoblaciones de dependientes emocionales, codependientes y bidependientes obtienen puntuaciones más bajas estadísticamente significativas $(p<.0001)$ en relación al resto de submuestras, lo cual es aplicable a cada una de las subescalas respecto a la correspendiente subpoblación clínica. Estas diferencias se confirman respecto a la población de Adictos, así como en relación con la población general, así como en relación a los familiares no codependientes (véanse Tabla 7 y 8).

Se ha efectuado un análisis de comparación de medias, confirmándose los indicios de mayor patología en muestras clínicas en comparación con población general respecto a subpoblaciones de comparación (adictos y familiares no codependientes) y en relación a la población general.

El sentimiento de Interdependencia y de Autoengaño es característico de los Bidependientes y Dependientes Emocionales. Ambos comparten la incapacidad para darse cuenta de los efectos adversos de la relación y en general de todas las componentes negativas de la misma, descrita como Autoengaño.

Específicamente, se comprueba el perfil afectivo del Dependiente Emocional con presencia de Sentimientos negativos y una mayor Acomodación situacional con el comportamientos de adaptación pasiva al otro/a, falta de iniciativa con indiferencia e inedia ante la situación relacional vivida.

En las tres poblaciones clínicas los desajustes identitarios (Caracterosis) son característicos con mecanismos de identificación, con presencia de juegos de poder asociado al control y el dominio en la relación, así como con fronteras en las relaciones interpersonales.

Los Antecedentes familiares se presentan en las muestras de Bidependientes, Dependientes Emocionales y Adictos en mayor medida que en el resto, y, finalmente, los Codependientes presentan un perfil muy específico, constatándose diferencialmente en relación al resto de subpoblaciones clínicas, de comparación y población general una acusada tendencia al Heterocontrol con Focalización en el otro e Hipercontrol. Asimismo, presentan una marcada Sobreprotección con tendencia a la necesidad de ayudar al otro/a y a los demás, intentando resolver sus problemas. El mecanismo relacional de Heterocontrol se confirma que representa la principal seña identificativa de los codependientes. 
Tabla 7. Puntuaciones medias en la Escala General, en las subescalas y dimensiones del IRIDS-100 por subtipo de población

\begin{tabular}{|c|c|c|c|c|c|c|}
\hline Escala General, Subescalas & $\mathrm{DE}$ & CDP & BDP & Población & Adictos & Familiares no CDP \\
\hline \multicolumn{7}{|l|}{ y Dimensiones IRIDS-100 } \\
\hline Escala General & 2.564 & 2.887 & 2.700 & 3.318 & 3.121 & 3.676 \\
\hline \multicolumn{7}{|l|}{ Subescalas } \\
\hline Dependencia Emocional & 2.283 & 2.817 & 2.755 & 3.079 & 3.018 & 3.420 \\
\hline Codependencia & 2.816 & 2.603 & 3.096 & 3.244 & 2.955 & 3.474 \\
\hline Bidependencia & 2.631 & 3.095 & 2.380 & 3.656 & 3.247 & 3.864 \\
\hline \multicolumn{7}{|l|}{ Dimensiones } \\
\hline Triada Dependiente / Interdependencia & 2.489 & 3.132 & 2.532 & 3.412 & 3.195 & 3.815 \\
\hline Acomodación & 2.511 & 2.939 & 2.918 & 2.903 & 3.390 & 3.541 \\
\hline Autoengaño & 2.560 & 2.989 & 2.547 & 3.463 & 3.240 & 3.529 \\
\hline Sentimientos adversos & 2.247 & 2.697 & 2.644 & 3.189 & 2.894 & 3.682 \\
\hline Identidad / Caracterosis & 2.831 & 3.019 & 3.047 & 3.532 & 3.320 & 3.902 \\
\hline Antecedentes personales & 2.977 & 3.176 & 2.788 & 3.743 & 3.114 & 4.118 \\
\hline Triada Codependiente/ Heterocontrol & 2.608 & 2.432 & 2.678 & 3.125 & 2.908 & 3.359 \\
\hline
\end{tabular}

Nota: D.E.=Dependientes Emocionales; BDP=Bidependendientes; CDP=Codependientes

Tabla 8. Comparaciones de medias (ANOVA) y contrastes Post-hoc entre las subpoblaciones clínicas, de comparación y control en las Escalas y Dimensiones del IRIDS-100

\begin{tabular}{llc}
\hline Escala General, Subescalas & $F$ & Comparaciones Post-hoc \\
y Dimensiones IRIDS-100 & & \\
\hline Escala General & $41.113^{*}$ & DE $>$ BDP, CDP $>$ Adictos, P. General $>$ Fam. no CDP \\
Subescala Dependencia Emocional & $35.359^{*}$ & DE $>$ BDP, CDP $>$ Adictos, P. General $>$ Fam. no CDP \\
Subescala Codependencia & $31.825^{*}$ & CDP $>$ DE, BDP $>$ Adictos $>$ P. General $>$ Fam. no CDP \\
Subescala Bidependencia & $79.015^{*}$ & BDP $>$ DE $>$ CDP, Adictos $>$ P. General $>$ Fam. no CDP \\
Interdependencia & $49.940^{*}$ & DE, BDP $<$ CDP, Adictos $<$ G.Control $<$ Familiares no-CDP \\
Acomodación Situacional & $40.971 *$ & DE $<$ BDP, CDP, G.Control $<$ Adictos, Familiares no-CDP \\
Autoengaño & $51.025^{*}$ & BDP, DE $<$ CDP $<$ Adictos $<$ G.Control $<$ Familiares no-CDP \\
Sentimientos Negativos & $45.199 *$ & DE $<$ BDP,CDP,Adictos $<$ Población G. $<$ Familiares no-CDP \\
Caracterosis & $50.016^{*}$ & DE, CDP, BDP $<$ Adictos $<$ G.Control $<$ Familiares no-CDP \\
Antecedentes personales & $35.656^{*}$ & BDP, D.E., Adictos $<$ CDP $<$ G.Control $<$ Familiares no-CDP \\
Triada Codependiente / Heterocontrol & $31.825^{*}$ & CDP $<$ DE, BDP $<$ Adictos $<$ G.Control $<$ Familiares no-CDP
\end{tabular}

Nota: * $p<, 0001$

D.E. =Dependientes Emocionales, BDP =Bidependendientes, CDP =Codependientes, P. General = Población General, Fam. no CDP= Familiares no Codependientes.

\section{DISCUSIÓN}

En la actualidad las relaciones interpersonales se hallan sometidas a unas singularidades, presiones y simbolismos que las caracterizan, máxime ante unas condiciones relacionales de cambios en los estilos de expresión de los afectos y sentimientos (FreitasMagalhães, 2007; Greenberg, \& Goldman, 2008; Hicks, \& Hicks, 2008; Nussbaum, 2008), de ahí la conveniencia de evaluar de forma integral estas dependencias.
De acuerdo con los resultados obtenidos, conceptualizamos las Dependencias Relacionales (D.R.) como trastornos caracterizados por la manifestación de comportamientos adictivos en la relación interpersonal basados en una asimetría de rol y en una actitud dependiente en relación al sujeto del que se depende (Sirvent, 2000). Se incide sobre el patrón crónico de demandas afectivas frustradas, la disfuncionalidad de los vínculos, la desadaptación de las relaciones, la intensidad de los afectos, la asimetría de la relación y la necesidad cronificada del otro, como principales descriptores 
sintomáticos de una Dependencia Relacional o Sentimental. El miedo a la pérdida, a la soledad y/o al abandono contamina el vínculo afectivo y lo vuelve sumamente vulnerable y patológico. La mistificación, el autoengaño o los mecanismos de negación reestructuran cognitivamente nuestra percepción de las relaciones. La manifestación de sentimientos negativos unida a la inescapabilidad emocional cronifica un vínculo disfuncional, aunque adaptativo, para quien lo sufre por la propia distorsión cognitiva. Aún así, la base etiológica no se fundamenta en exclusividad sobre lo caracteriológico o lo relacional, sino que las representaciones sociales sobre el amor e incluso indicadores macrocontextuales afectan a los desórdenes personales, tal y como hemos expuesto en los apartados anteriores. La disfuncionalidad, la saliencia y la intensidad de los afectos toxicofílicos son elementos clave de esa vinculación a los otros que se convierte en dependencia (véase Moral, \& Sirvent, 2008, 2009; Sirvent, 2000).

En cualquier caso, anticipemos que los autores de este artículo somos muy críticos con el exceso de diagnósticos que salpican la psicopatología (Frances, 2014) quedando lejos de nuestra intención añadir una afección más al ya largo y prolijo catálogo de enfermedades. Sin embargo, el constructo analizado posee entidad y razón de ser por el impacto sociosanitario del mismo. Nada menos que un $8.66 \%$ de la población general (grupo control) tiene un nivel notable de dependencia relacional. Si nos ceñimos a poblaciones clínicas el porcentaje se dispara (hasta un 65\% de mujeres bidependientes con pareja). Añadamos que es un fenómeno silencioso que no se detecta fácilmente por la natural tendencia de quienes lo padecen a ocultarlo o malinterpretarlo, emergiendo a través de síntomassatélite como la ansiedad o la depresión. Vale la pena, pues, analizar y profundizar en el fenómeno y como paso previo ofrecer un instrumento sólido, fiable y útil para evaluarlo. Eso es lo que se ha pretendido con el IRIDS-100, de modo que se ha verificado la utilidad clínica del instrumento mediante aplicaciones seriadas longitudinales, confirmando los profesionales la practicidad del mismo desde una perspectiva tanto evaluativa como terapéutica. Esta doble aplicatividad servirá para que tanto investigadores clínicos como psicoterapeutas se puedan beneficiar del instrumento.

Para concluir, señalemos que existe una interesante puerta abierta a la indagación prospectiva del fenómeno dado su interés clínico y su porvenir investigador (véase Moral, \& Sirvent, 2014). Proponemos avanzar en la investigación sobre el fenómeno en sí y sobre los factores asociados que marcan un trazado prospectivo de notable validez clínica-investigadora.

\section{REFERENCIAS}

Bartholomew, K., \& Horowitz, L. M. (1991). Attachment styles among young adults: A test of a four-category model. Journal of Personality and Social Psychology, 61, 226-244. DOI: 10.1037/00223514.61.2.226

Bornstein, R. F., \& Huprich, S. K. (2006). Construct Validity of the Relationship Profile Test: Three-Year Retest Reliability and Links With Core Personality Traits, Object Relations, and Interpersonal Problems. Journal of Personality Assessment, 86(2), 162-171. DOI: 10.1207/s15327752jpa8602_05

Carretero-Dios H., \& Pérez, C. (2007). Normas para el desarrollo y revisión de estudios instrumentales: consideraciones sobre la selección de tests en la investigación psicológica. International Journal of Clinical and Health Psychology, 7(3), 863-882.

Coleman, E. (1987). Marital and relationship problems among chemically dependent and codependent relationships. Journal of Chemical Dependency Treatment, 1(1), 39-59. DOI: 10.1300/J034v01n01_04

Comrey, A. L. (1988). Factor-analytic methods of scale development in personality and clinical psychology. Journal of Consulting and Clinical Psychology, 56, 754-761.

Cook D. R. (1987). Self-identified addictions and emotional disturbances in a sample of college students. Psychology of Addictive Behaviors, 1, 55-61.

Cortina, J. M. (1993). What is coefficient alpha? An examination of theory and applications. Journal of Applied Psychology, 78, 98-104.

Ellis, B., Simpson, J. A., \& Campbell, L. (2002). Trait-Specific Dependence in Romantic Relationships. Journal of Personality 70, 611-659. DOI: 10.1111/1467-6494.05019

Fausel, D. F. (1988). Helping the helper heal: Co-dependency in helping professionals. Journal of Independent Social Work, 3(2), 35-45. DOI: 10.1300/J283v03n02_04

Frances A. (2014). ¿Somos todos enfermos mentales?: manifiesto contra los abusos de la psiquiatría. Barcelona: Ariel.

Freimuth, M., Waddell, M., Stannard, J., Kelley, S., Kipper, A., Richardson, A., \& Szuromi, I. (2008). Expanding the scope of dual diagnosis and co-addictions: Behavioral addictions. Journal of Groups in Addiction \& Recovery, 3, 137-160.

Freitas-Magalhães, A. (2007). The Psychology of Emotions: The allure of human face. Oporto: University Fernando Pessoa Press.

Greenberg, L. S., \& Goldman, R. N. (2008). Emotion-focused couples therapy for couples. New York: Guilford Press. 
Hands, M., \& Dear, G. (1994). Codependency: a critical review. Drug and Alcohol Review, 13(4), 437-45.

Hatfield, E., \& Sprecher, S. (1986). Measuring passionate love in intimate relationships. Journal of Adolescence, 9, 383-410.

Hernández, R., Fernández, C., \& Baptista, P. (1998). Metodología de la Investigación. México, D. F.: McGraw-Hill Interamericana.

Hicks, E., \& Hiscks, J. (2008). El increíble poder de las emociones: atrévete a dejarte guiar por los sentimientos. Barcelona: Urano.

Hirschfeld, R. M. A., Klerman, G. L., Harrison, M., Gough, H. G., Barret, J, Korchin, S. J., \& Chodoff, P. (1977). A measure of interpersonal dependency. Journal of Personality Assessment, 41(6), 610-618.

Hughes-Hammer C., Martsolf, D. S., \& Zeller, R. A . (1998). Depression and codependency in women. Archives of Psychiatric Nursing, 12, 326334. DOI: 10.1016/S0883-9417(98)80046-0.

Irwin, H. J. (1995). Codependence, narcissism, and childhood trauma. Journal of Clinical Psychology, 51(5), 658-665. DOI 10.1002/1097-4679

Jiménez Burillo, F., Sangrador, J. L., Barrón, P. y Yela, C. (1995). Análisis psicosocial sobre el comportamiento amoroso de los españoles. Madrid: C.I.S. Estudio n 2157.

Krueger R. B., \& Kaplan, M. S. (2001). The paraphilic and hypersexual disorders: An overview. Journal of Psychiatric Practice, 7, 391-403.

Kuzma, J. M., \& Black, D. W. (2008). Epidemiology, prevalence, and natural history of compulsive sexual behavior. Psychiatric Clinics of North America, 31, 601-611. DOI: 10.1016/j.psc.2008.06.005

MacLaren, V. V., \& Best L. A. (2010). Multiple addictive behaviors in young adults: Student norms for the Shorter PROMIS. Questionnaire. Addictive Behaviors, 35, 252-255.

Madey, S. F., \& Rodgers, L. (2009). The Effect of Attachment and Sternberg's Triangular Theory of Love on Relationship Satisfaction. Individual Differences Research, 7(2), 76-84.

Marks, A. D. G., Blore, R. L., Hine, D. W., \& Dear, G. (2012). Development and validation of a revised measure of codependency. Australian Journal of Psychology, 64(3), 119-127. DOI: 10.1111/j.17429536.2011.00034.x

Masuda, M. (2003). Meta-analyses of love scales: Do various love scales measure the same psychological constructs? Japanese Psychological Research, 45(1), 25-37. DOI: 10.1111/1468-5884.00030

Moral, M. V., \& Sirvent, C. (2008). Dependencias sentimentales o afectivas: etiología, clasificación y evaluación. Revista Española de Drogodependencias, 33(2), 145-167.

Moral, M. V., \& Sirvent, C. (2009). Dependencia afectiva y género: Perfil sintomático diferencial en dependientes afectivos españoles. Revista Interamericana de Psicología, 43(2), 230-240.

Moral, M. V., \& Sirvent, C. (2014). Objetivos psicoterapéuticos e intervención integral en Dependencias Sentimentales. Revista Española de Drogodependencias, 39(3), 38-56.
Moral, M. V., García, A., Cuetos, G., \& Sirvent. C. (2017). Violencia en el noviazgo, dependencia emocional y autoestima en adolescentes y jóvenes españoles. Revista Iberoamericana de Psicología y Salud, 8(2), 96-107. DOI: 10.23923/j.rips.2017.08.009

Nussbaum, M.C. (2008). Paisajes del pensamiento: La inteligencia de las emociones. Barcelona: Paidós.

Pérez, A., \& Delgado, D. (2003). La codependencia en familias de consumidores y no consumidores de drogas: Estado del arte y construcción de un instrumento. Psicothema, 15(3), 381-387.

Roehling, P. V., \& Gaumond, E. (1996). Reliability and validity of the codependence questionnaire. Alcoholism Treatment Quarterly, 14, 85-95. DOI: 10.1300/J020V14N01_08

Rubin, Z. (1970). Measurement of romantic love. Journal of Personality and Social Psychology, 16, 265-273. DOI: 10.1037/h0029841

Scharfe, E., \& Bartholomew, K. (1994). Reliability and stability of adult attachment patterns. Review Personal Relationships, 1, 23-43. DOI: 10.1111/j.1475-6811.1994.tb00053.x

Scott, P. (1997). Hacia una psicología del amor. Barcelona: Emecé Editores.

Sirvent, C. (1999), La bidependencia como dependencia relacional. Editorial web lasDrogas.info. http://www.lasdrogas.info/opiniones/19/la-bidependencia-comodependencia-relacional.html

Sirvent, C. (2000). Las dependencias relacionales dependencia emocional, codependencia y bidependencia. Libro del I Symposium Nacional sobre Adicción en la Mujer (pp. 164-215). Madrid: Instituto de la Mujer y Fundación Instituto Spiral.

Sirvent, C., \& Moral, M. V. (2007). Presentación del TDS- 100, test de Dependencias Sentimentales de Sirvent y Moral. Anales de Psiquiatría, 23(3), 94-95.

Sirvent C., Moral M. V., Blanco P., \& Palacios L. (2004.) Estudio sobre Interdependencia Afectiva en la Población General. Congreso de La Sociedad Española de Toxicomanías. Santiago de Compostela.

Snook, S. C., \& Gorsuch, R. L. (1989). Component analysis versus common factor analysis: a Monte Carlo study. Psychological Bulletin, 106, 148154.

Spann, J., \& Fischer, L. (1991). Measuring codependency. Alcoholism Treatment Quarterly, 8(1), 87-100. DOI: 10.1300/J020V08N01_06

Sternberg, R. J. (2013). Searching for love. Psychologist, 26(2), 98-101.

Sussman S., Lisha N., \& Griffiths M. (2010). Prevalence of the Addictions: A Problem of the the Majority or the Minority? National Institute of Health (NIH). Evaluation \& the Health Professions, 34(1), 3-56. DOI: $10.1177 / 0163278710380124$

Timmreck, T. C. (1990). Overcoming the loss of a love: preventing love addiction and promoting positive emotional health. Psychological Reports, 66(2), 515-28. DOI: 10.2466/pr0.1990.66.2.515 